\title{
Myogenic Differentiation Potential of Mesenchymal Stem Cells Derived from Fetal Bovine Bone Marrow
}

\section{Lucas Hidenori Okamura, Paloma Cordero, Jaime Palomino, Victor Hugo} Parraguez, Cristian Gabriel Torres \& Oscar Alejandro Peralta

To cite this article: Lucas Hidenori Okamura, Paloma Cordero, Jaime Palomino, Victor Hugo Parraguez, Cristian Gabriel Torres \& Oscar Alejandro Peralta (2018) Myogenic Differentiation Potential of Mesenchymal Stem Cells Derived from Fetal Bovine Bone Marrow, Animal Biotechnology, 29:1, 1-11, DOI: 10.1080/10495398.2016.1276926

To link to this article: https://doi.org/10.1080/10495398.2016.1276926

曲 Published online: 07 Mar 2017.

Submit your article to this journal $₫$

Џ Article views: 240

View Crossmark data ¿

Citing articles: 6 View citing articles $\sqsubset$ 


\title{
Myogenic Differentiation Potential of Mesenchymal Stem Cells Derived from Fetal Bovine Bone Marrow
}

\author{
Lucas Hidenori Okamuraa, ${ }^{\mathrm{a},}$ Paloma Cordero ${ }^{\mathrm{b}}$, Jaime Palomino ${ }^{\mathrm{b}}$, Victor Hugo Parraguez ${ }^{\mathrm{c}}$, Cristian Gabriel Torres ${ }^{\mathrm{d}}$ \\ and Oscar Alejandro Peraltab,e \\ aDepartamento de Apoio, Produção e Saúde Animal, Faculdade de Medicina Veterinária, Universidade Estadual Paulista "Júlio de Mesquita \\ Filho", Araçatuba, São Paulo, Brasil; 'Departamento de Fomento de la Producción Animal, Facultad de Ciencias Veterinarias y Pecuarias, \\ Universidad de Chile, Santiago, Chile; 'Departamento de Ciencias Biológicas, Facultad de Ciencias Veterinarias y Pecuarias, Universidad de \\ Chile, Santiago, Chile; 'Departamento de Ciencias Clínicas, Facultad de Ciencias Veterinarias y Pecuarias, Universidad de Chile, Santiago, Chile; \\ 'Department of Biomedical Sciences and Pathobiology, Virginia-Maryland Regional College of Veterinary Medicine, Virginia Tech, Blacksburg, \\ Virginia, USA
}

\begin{abstract}
The myogenic potential of bovine fetal MSC (bfMSC) derived from bone marrow (BM) remains unknown; despite its potential application for the study of myogenesis and its implications for livestock production. In the present study, three protocols for in vitro myogenic differentiation of bfMSC based on the use of DNA methyltransferase inhibitor 5-Aza-2'-deoxycytidine (5-Aza), myoblast-secreted factor Galectin-1 (Gal-1), and myoblast culture medium SkGM-2 BulletKit were used. Plastic-adherent bfMSC were isolated from fetal BM collected from abattoir-derived fetuses. Post-thaw viability analyses detected $85.6 \%$ bfMSC negative for propidium iodine (PI). Levels of muscle regulatory factors (MRF) MYF5, MYF6, MYOD, and DES mRNA were higher $(\mathrm{P}<0.05)$ in bfMSC cultured under $100 \mu \mathrm{M}$ of 5-Aza compared to 1 and $10 \mu \mathrm{M}$. Treatment of bfMSC with $10 \mu \mathrm{M}$ of 5-Aza resulted in down-regulation of MYOD mRNA (Days 7 to 21) and up-regulation of MYF6 (Day 7), MYF5, and DES mRNA (Day 21). Gal-1 and SkGM-2 BulletKit induced sequential downregulation of early MRF (MYF5) and up-regulation of intermediate (MYOD) and late MRF (DES) mRNA. Moreover, DES and MYF5 were immunodetected in differentiated bfMSC. In conclusion, protocols evaluated in bfMSC induced progress into myogenic differentiation until certain extent evidenced by changes in MRF gene expression.
\end{abstract}

\section{KEYWORDS}

Bone marrow; cattle; mesenchymal stem cells; myogenesis

\section{Introduction}

Mesenchymal stem cells (MSC) are multipotent progenitor cells defined by their ability to self-renew and give rise to differentiated progeny. MSC can be isolated from several tissue sources including bone marrow (BM), based on their ability to adhere to plastic when plated in monolayer culture (1). Thereafter, multipotent MSC can differentiate in vitro into mature cells of mesenchymal lineages including osteocytes, chondrocytes and adipocytes (2). It has been shown that under certain conditions, MSC are also able to differentiate into other mesodermal tissues including skeletal muscle (3).

The myogenic potential of MSC has been previously explored by in vivo engraftment experiments in mice $(4,5)$. Moreover, the myogenic potential of MSC has also been explored in vitro under the effect of different exogenous factors. The DNA methyltransferase inhibitor, 5-Aza-2'-deoxycytidine (5-Aza) has been used as a myogenic inductor on mice MSC, through the stochastic hypomethylation of random DNA residues leading to the activation of Desmin (DES), a muscle-specific intermediate filament (IF) protein $(6,7)$. Galectin-1 (Gal-1) is a $\beta$-galactoside-binding protein secreted by myoblasts that has also been used as a myogenic inducer in human fetal MSC (hfMSC) (8). The hfMSC treated with Gal-1 acquired muscle phenotype, formed long multinucleated fibers, and expressed DES and myosin (MYO) via the activation of several muscle regulatory factors (MRF) (8). Furthermore, the commercially available myoblast culture medium SkGM-2 BulletKit, which contains myogenic additives including dexamethasone, epidermal growth factor (EGF), and fetuin has also been previously evaluated in human MSC (9). The effect of SkGM-2 BulletKit led to high proliferation rates and increased expression of myosin heavy chain (MYH) in MSC derived from adipose tissue (9).

Myogenesis during embryogenesis is a wellorchestrated and highly complex process that involves

CONTACT Oscar Alejandro Peralta operalta@uchile.cl Departamento de Fomento de la Producción Animal, Facultad de Ciencias Veterinarias y Pecuarias, Universidad de Chile, Santiago, 8820808, Chile. 
sequential activation of several factors associated to cell proliferation and differentiation. The MRF, including myogenic factor 5 and 6 (MYF5 and MYF6), myogenic determining factor (MYOD), and myogenin (MYOG) are a family of basic helix-loop-helix transcription factors that have been shown to regulate muscle development (10). MYF5 is an early marker of differentiation that is up-regulated during the early stages of myogenesis, acting as a promoter of multiple muscle-specific genes and controlling the fusion of multinucleated myofibers $(11,9)$. MYF6 regulates the final differentiation of myotubes and is regarded as the principal factor influencing skeletal muscle phenotype (12). MYOD gene regulates differentiation of the muscle and increases the transcriptional activation of various downstream target genes including MYOG (13). The transcriptional activity of MYOD is regulated by DNA methylation of the transcriptional region $(14,15)$. MYOG is a critical transcription factor in myogenesis that regulates expression of most of the muscle-specific genes and is essential for the terminal differentiation of committed myoblasts (16). Thereafter, in the mature muscle, DES forms a sarcoplasmic network that encircles $\mathrm{Z}$ disks to connect and binds them to the sarcoplasmic membrane and the nuclear lamina (17).

In vitro studies performed in our laboratory have demonstrated the multipotency of progenitor bovine MSC isolated from fetal BM (bfMSC), including its capacity to differentiate into osteocytes, chondrocytes, adipocytes (18), hepatocytes, and neurons (19). Considering that differentiation into musculoskeletal lineages is one feature of MSC; the in vitro differentiation of bfMSC constitutes an excellent experimental model that resembles myogenesis of progenitor MSC during bovine muscle fetal development. Together, myocytes, adipocytes, and fibroblasts constitute the basic structure of skeletal muscle and because the majority of these cells in fetal muscle derived from the same pool of MSC (20), defining mechanisms regulating MSC differentiation is crucial in order to improve carcass quality and meat production efficiency for livestock industry. The aim of the present study was to evaluate the myogenic differentiation potential of bfMSC using three protocols based on the use of 5-Aza, Gal-1, and SkGM-2 BulletKit.

\section{Materials and methods}

\section{Isolation, culture, and freezing of bfMSC}

All procedures have been approved by the Bioethical Committee of the National Commission for Scientific and Technology Research from Chile (Fondecyt). Bone marrow was aspirated from bovine fetuses $(n=9 ; 7-8$ months of gestation) collected at a local abattoir. Animals slaughtered were from different farms and breeds and no health records were available. Samples of muscle (Longissimus dorsi) were collected from the same fetuses and used as positive controls. The marrow was drawn from femoral marrow cavity into syringes containing high glucose Dulbecco's Modified Eagle Medium (DMEM, Gibco, Grand Islands, NY, USA) supplemented with $10 \%$ fetal bovine serum (FBS), $1000 \mathrm{IU}$ heparin, $100 \mathrm{IU} / \mathrm{mL}$ penicillin, and $100 \mu \mathrm{g} / \mathrm{mL}$ streptomycin. BM samples from three fetuses were pooled and washed twice with phosphate-buffered saline (PBS) and twice with DMEM. Then, cells were plated in DMEM (high glucose) supplemented with $10 \%$ FBS, $100 \mathrm{IU} / \mathrm{mL}$ penicillin, $100 \mu \mathrm{g} / \mathrm{mL}$ streptomycin and $0.25 \mu \mathrm{g} / \mathrm{mL}$ amphotericin B. Cells were incubated at $38^{\circ} \mathrm{C}$ in a humidified atmosphere containing $5 \%$ $\mathrm{CO}_{2}$. After two days, non-adherent cells were removed by changing the culture medium. Following the initial two days, the medium was changed every two to three days. After 3 to 4 passages, cells were gently harvested when $90 \%$ confluent using $0.25 \%$ trypsin in $0.1 \%$ EDTA. Following determination of cell viability, cells were used to initiate experiments. Approximately $1 \times 10^{6}$ bfMSC were resuspended in $1 \mathrm{~mL}$ of freezing media consistent in DMEM (high glucose) supplemented with $10 \%$ FBS, $10 \%$ dimethyl sulfoxide (DMSO), $100 \mathrm{IU} / \mathrm{mL}$ penicillin, $100 \mu \mathrm{g} / \mathrm{mL}$ streptomycin, and $0.25 \mu \mathrm{g} / \mathrm{mL}$ amphotericin $\mathrm{B}$ and pipetted into cryogenic vials. The bfMSC were frozen at $-80^{\circ} \mathrm{C}$ for 24 hours and then placed in liquid $\mathrm{N}_{2}$ at $-196^{\circ} \mathrm{C}$. Cells were thawed by placing cryogenic vials in water at $37^{\circ} \mathrm{C}$ for 3 minutes.

\section{Flow cytometry}

Determination of post-thaw viable cells was estimated by quantification of propidium iodide (PI)-negative bfMSC using flow cytometry. After thawing, approximately $10 \times 10^{5}$ cells were centrifuged and incubated in $1 \mu \mathrm{g} / \mathrm{mL}$ PI (Molecular probes, Invitrogen, Oregon, USA) in PBS for 5 minutes. After three washes on PBS, the pellet was resuspended on IsoFlow buffer and analyzed (three replicates) using a Gallios Flow Cytometer (Beckman Coulter, Brea, CA, USA) using a 606-635 nm laser light. The threshold for negative events was set on the first decade of fluorescence level histogram. Negative procedural control corresponded to unfrozen bfMSC.

\section{Myogenic differentiation protocols}

\section{5-Aza}

The effect of various concentrations of 5-Aza (1, 10,

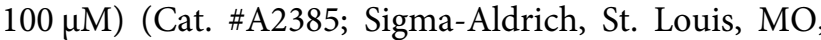


USA) was first analyzed on myogenic differentiation of bfMSC. Cells $\left(5 \times 10^{4} / \mathrm{cm}^{2}\right)$ were plated in T-25 culture dishes either in control (three replicates) or differentiation (three replicates) medium and cultured for a 21-Day experiment with the medium being changed every 2 days. Control medium consisted of DMEM (high glucose) supplemented with $10 \% \mathrm{FBS}, 100 \mathrm{IU} / \mathrm{mL}$ penicillin, $100 \mu \mathrm{g} / \mathrm{mL}$ streptomycin and $0.25 \mu \mathrm{g} / \mathrm{mL}$ amphotericin B. The myogenic differentiation in bfMSC was evaluated on Days 0 and 21. Considering that $10 \mathrm{mM}$ 5-Aza allowed high cell survival and myogenic gene expression, bfMSC were cultured under this concentration for further time-response experiments using the same conditions described previously. Samples were obtained at Days 0, 7, 14, and 21 and analyzed for myogenic gene expression by quantitative-PCR (Q-PCR). The level of myogenic differentiation was also analyzed at Day 21 of differentiation by immunodetection of DES and MYF5 protein.

\section{Gal-1}

Cells $\left(2-5 \times 10^{3} / \mathrm{cm}^{2}\right)$ isolated from 3 fetuses were seeded in T-25 culture dishes in control (three replicates) or differentiation (three replicates) medium. Control medium consisted of DMEM (high glucose) supplemented with $10 \%$ FBS, $100 \mathrm{IU} / \mathrm{mL}$ penicillin, $100 \mu \mathrm{g} / \mathrm{mL}$ streptomycin, and $0.25 \mu \mathrm{g} / \mathrm{mL}$ amphotericin B. Differentiation medium was adapted from a previously reported protocol (8) and consisted in control medium supplemented with $10 \%$ FBS and $100 \mathrm{nM}$ Gal-1 (recombinant human, Cat. \#1152-GA; R\&D systems, Minneapolis, MN, USA). Cells were cultured for a 12-Day experiment with the medium being changed every 2 days. Samples were obtained at four-day intervals and analyzed for myogenic gene expression by Q-PCR. The level of myogenic differentiation was also analyzed at Day 12 of differentiation by immunodetection of DES and MYF5 protein.

\section{SkGM-2 BulletKit}

Induction of myogenic differentiation was performed using an adapted protocol from a previously published report (9). Cells $\left(3 \times 10^{3} / \mathrm{cm}^{2}\right)$ at passages 3 to 5 were plated in T-25 culture dishes either in control or differentiation medium. Control medium consisted of DMEM (high glucose) supplemented with 10\% FBS, $100 \mathrm{IU} / \mathrm{mL}$ penicillin, $100 \mu \mathrm{g} / \mathrm{mL}$ streptomycin, and $0.25 \mu \mathrm{g} / \mathrm{mL}$ amphotericin B. Differentiation medium consisted in SkGM-2 BulletKit (Lonza, Walkersville, MD, USA). This cell culture medium contains SkBM Basal Medium supplemented with EGF, fetuin, 10\% FBS, dexamethasone, insulin, and gentamicin/amphotericin. The precise composition is only known to the manufacturer. Cells were cultured for a 21-Day experiment with the medium being changed every 2 days. Samples were obtained at seven-day intervals and analyzed for myogenic gene expression by Q-PCR. The level of myogenic differentiation was also analyzed at Day 21 of differentiation by immunodetection of DES and MYF5 protein.

\section{RNA extraction and CDNA synthesis}

Approximately $3 \times 10^{5}$ bfMSC and muscle samples were collected and immediately fixed in RLT buffer (Qiagen, Incorporated, Valencia, CA, USA). Total RNA was extracted using RNeasy Mini kit (Qiagen) according to the manufacturing's instructions. The concentration and purity of the RNA in each sample were determined using Qubit RNA assay kit (Thermo Fisher Scientific, Waltham, MA, USA). Total RNA was eluted in $30-50 \mu \mathrm{L}$ of RNase free water. Samples were subjected to RT-PCR using a Brilliant II SYBR Green RT-PCR kit (Agilent Technologies, Santa Clara, CA, USA). The reaction protocol consisted of incubation for 5 minutes at $25^{\circ} \mathrm{C}, 15$ minutes at $42^{\circ} \mathrm{C}, 5$ minutes at $95^{\circ} \mathrm{C}$, and hold at $4^{\circ} \mathrm{C}$ using a DNA engine PCR thermocycler (Bio-Rad Laboratories, Hercules, CA, USA).

\section{Quantitative-PCR}

Samples were analyzed for housekeeping $\beta$-ACTIN and GAPDH, and myogenic genes MYF5, MYF6, MYOD, $M Y O G$, and DES expression by Q-PCR. The $\beta$-ACTIN was selected for housekeeping gene based on previous analyses from our and others laboratories $(18,19,9)$ that detected high stability during MSC culture. Realtime PCR primers were designed using PrimerExpress software (Applied Biosystems Incorporated, Foster City, CA, USA; Table 1). Equivalence of amplification

Table 1. Sequence of primers used for Q-PCR analysis.

\begin{tabular}{|c|c|c|c|}
\hline Gene & Sense & Antisense & Accession number \\
\hline MYF5 & CAGGAATGCCATCCGCTACA & GGCAATCCAGGTTGCTCTGA & NM_174116.1 \\
\hline MYF6 & GGTGGACCCCTTCAGCTACAG & TGCTTGTCCCTCCTTCCTTGG & NM_181811.1 \\
\hline MYOD & CGACTCGGACGCTTCCAGT & GATGCTGGACAGGCAGTCGA & NM_001040478.2 \\
\hline MYOG & GTGCCCAGTGAATGCAGCTC & GTCTGTAGGGTCCGCTGGGA & NM 001111325.1 \\
\hline DESMIN & GGACCTGCTCAATGTCAAGA & GGAAGTTGAGGGCAGAGAAG & NM_001081575.1 \\
\hline$\beta-A C T I N$ & CGCACCACTGGCATTGTCAT & TCCAAGGCGACGTAGCAGAG & NM_001033618.1 \\
\hline GAPDH & СCTTCATTGACCTTCACTACATGGTCTA & TGGAAGATGGTGATGGCCTTTCCATTG & NM_001034034.2 \\
\hline
\end{tabular}


efficiencies among all primer-probe sets was confirmed using serial 3-fold dilutions of differentiated bfMSC cDNA. Each RT-PCR reaction $(10 \mu \mathrm{L})$ contained the following: 2X Brilliant II SYBR Green QPCR master mix $(5 \mu \mathrm{L})$, target forward primer $(200 \mathrm{nM})$, target reverse primer $(200 \mathrm{nM})$, cDNA synthesis reaction $(1 \mu \mathrm{L})$, and nuclease-free PCR-grade water to adjust final volume. The PCR amplification was carried out in Eco Real-Time PCR System (Illumina Incorporated, San Diego, CA, USA). Thermal cycling conditions were $95^{\circ} \mathrm{C}$ for 10 minutes, followed by 40 repetitive cycles at $95^{\circ} \mathrm{C}$ for 30 seconds, and $60^{\circ} \mathrm{C}$ for 1 minute. The relative quantification of the target gene expression across treatment was evaluated using the comparative $\Delta \Delta \mathrm{CT}$ method. The CT value was determined by subtracting $\beta$-ACTIN CT value from the target CT value of the sample. Calculation of $\Delta \Delta \mathrm{CT}$ involved using target gene expression on Day 0 (sample with the highest CT value or lowest target expression) as an arbitrary constant to subtract from all other CT sample values. Samples of fetal muscle were used as positive control.

\section{Immunofluorescence}

Differentiated bfMSC were cultured in $35-\mathrm{mm}$ dishes, fixed in a $4 \%$ paraformaldehyde (PAF) for 10 minutes and stored at $4{ }^{\circ} \mathrm{C}$ under PBS. Cells were then washed twice in PBS twice and blocked in donkey serum (Sigma-Aldrich) for 30 minutes at RT. Cells were incubated overnight at $4{ }^{\circ} \mathrm{C}$ with goat polyclonal anti-DES (1:50, Cat.\# SC-7559; Santa Cruz Biotechnology, Santa Cruz, CA, USA) or goat polyclonal anti-MYF5 (1:50, Cat.\# SC-31946; Santa Cruz Biotechnology) diluted in donkey serum. After three washes with PBS, cells were incubated with donkey anti-goat IgG conjugated to FITC (1:200 in donkey serum). Then, cells were again washed three times in PBS and mounted under coverslips in a solution containing 4',6-diamidino-2phenylindole (DAPI, Santa Cruz Biotechnology, Santa Cruz, CA, USA). Samples were examined under epifluorescence and the results captured by digital photomicroscopy (Olympus, Tokyo, Japan).

\section{Data analysis}

Values of PI-negative cells and gene expression from three different replicates were transferred to a spreadsheet and then analyzed using Infostat software (Cordoba, Argentina). Data was normalized to logarithmic scale in base 10 for normality and mean values for each replicate were compared by one-way ANOVA. Gene expression values between days of culture and between treatments and controls were analyzed using Duncan's multiple comparison test $(\mathrm{P}<0.05)$.

\section{Results}

Isolation of bfMSC from BM was performed based on the capacity for plastic attachment under standard culture conditions that included DMEM media supplemented with 10\% FBS. Colonies of fibroblast-like cells attached to the plastic were visualized at Days 5-6 after seeding. Cells exhibited characteristic spindle shape and polygonal morphology. Post-thaw viability analyses detected $85.6 \%$ bfMSC negative for PI versus $91.1 \%$ $(\mathrm{P}>0.05)$ in the negative control cells (Fig. 1).

Initial analyses were performed in bfMSC and fetal muscle samples to quantify $\beta$-ACTIN and GAPDH mRNA levels using Q-PCR. Analyses detected $\beta$-ACTIN and GAPDH Ct values of 14.1 and 15.7 in bfMSC and of 26.3 and 32.9 in fetal muscle, respectively. These values suggested that mRNA levels of these housekeeping genes are vastly different in bfMSC compared to muscle. Moreover, analyses reported previously $(18,19,9)$ indicated that $\beta$-ACTIN possess higher stability during MSC culture suggesting its suitability for relative gene expression determination in MSC studies.

Furthermore, the myogenic potential of bfMSC was investigated under the in vitro effect of 5-Aza during a 21-day culture period. We first evaluated dose-response effect using 1,10 , and $100 \mu \mathrm{M}$ of 5-Aza and determined cell morphology changes and relative mRNA levels of various MRF at Days 0 and 21. Supplementation of $100 \mu \mathrm{M}$ of 5-Aza for 21-day period induced formation of cell projections and development of intricate cell interactions (Fig. 2A). bfMSC cultured under the effect of $100 \mu \mathrm{M}$ of 5-Aza expressed higher mRNA levels of MYF5, MYOD, and DES (4.6-, 25.8-, and 22.3-fold relative to Day 0, respectively; $\mathrm{P}<0.05)$ at Day 21 of culture (Fig. 2B). Moreover, relative expression of MYF6 was increased (4.3-fold relative to Day 21 control, $\mathrm{P}<0.05$ ) in bfMSC exposed to $100 \mu \mathrm{M}$ of 5-Aza for 21 days. In contrast, in this experimental model supplementation of 1 and $10 \mu \mathrm{M}$ of 5 -Aza had no effects on relative expression of selected MRF. Despite supplementation of $100 \mu \mathrm{M}$ of 5-Aza induced significant effect on MRF relative expression; high cell mortality and low cell adhesion was observed in bfMSC cultures under the effect of this concentration, suggesting cytotoxicity (Fig. 2A). Thus, for time-response experiments we decided to use a lower concentration of $10 \mu \mathrm{M}$ of 5-Aza. As presented previously, bfMSC exposed to $10 \mu \mathrm{M}$ of 5-Aza underwent progressive cell morphology changes that included formation of cell projections and development of intricate cell interactions during the 

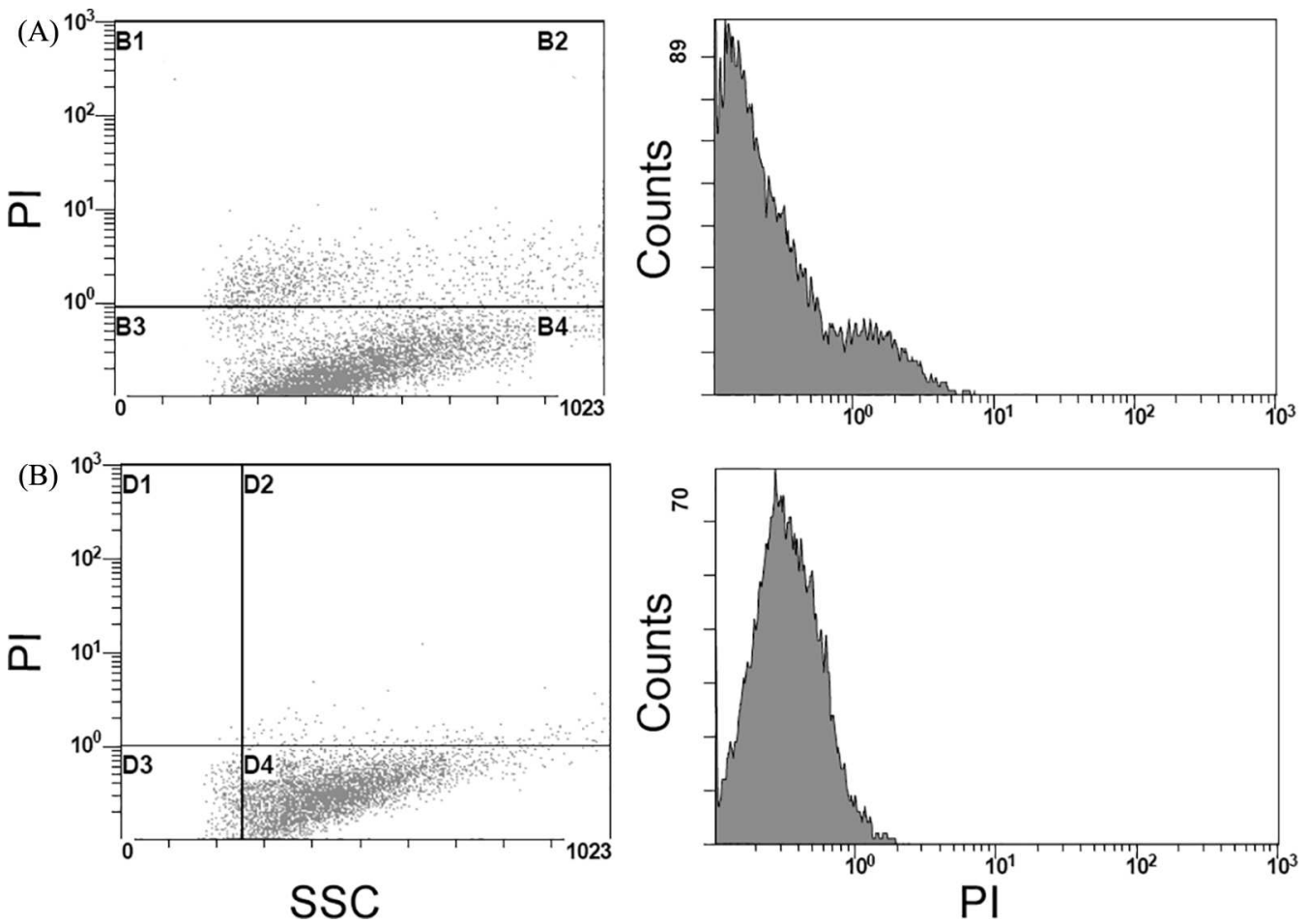

Figure 1. Flow-cytometric analysis of post-thawed bfMSC stained with propidium iodine to determine viability percentage. (A) Average percentage of propidium iodine-negative cells corresponded to $85.6 \%$. (B) Negative procedural control corresponded to unfrozen bfMSC. The threshold for negative events was set on the first decade of fluorescence level. Abbreviations: PI, Propidium lodine; SSC, Side Scatter.

21-day experiment (Fig. 2C). Observation of bfMSC cultures indicated no effect on cell mortality or adhesion to plastic. Moreover, after 7 days of differentiation, bfMSC increased MYF6 mRNA levels (1.5-fold Day-21 control, $\mathrm{P}<0.05)$ (Fig. 2D). In contrast, MYOD mRNA levels decreased from Day 7 (0.25-fold) to Day 21 (0.22-fold). At Day 21 of differentiation, DES and MYF5 mRNA levels were up-regulated (3.5- and 3-fold relative to Day $0, \mathrm{P}<0.05)$ in bfMSC cultured under $10 \mu \mathrm{M}$ of 5-Aza.

Culture of bfMSC under the effect of $100 \mathrm{nM}$ of Gal1 induced organization of cells into striated orientation pattern (Fig. 3A). The bfMSC cultured under the effect of Gal-1 decreased $(\mathrm{P}<0.05)$ MYF5 mRNA levels from Day 4 (0.26-fold) to Day 12 (0.32-fold) compared to Day 0 (Fig. 3B). In contrast, levels of MYOD, MYOG, and DES mRNA were up-regulated (4.7-, 4.8-, and 3.4-fold relative to Day 0, respectively; $\mathrm{P}<0.05)$ at Day 12 of culture. Culture of bfMSC in SkGM-2 BulletKit media resulted in subtle changes in cell morphology and distribution compared to bfMSC cultured in control media (Fig. 4A). Relative expression of MYF5 decreased $(\mathrm{P}<0.05)$ from Day $7(0.27$-fold) to Day 21 (0.27-fold) compared to Day 0 (Fig. 4B). In contrast, mRNA levels of DES increased $(\mathrm{P}<0.05)$ from Day 7 (4.7-fold) to Day 21 (2-fold) compared to Day 0. Levels of mRNA of MYOD were up-regulated at Day 7 (2.9fold relative to Day 0, $\mathrm{P}<0.05)$ and at Day 21 (2.7-fold relative to control Day $21, \mathrm{P}<0.05)$. Moreover, DES and MYF5 proteins were immunodetected in differentiated bfMSC at days 21 and 12 in presence of 5-Aza and Gal-1, respectively, and at day 21 of culture in SkGM-2 BulletKit (Fig. 5). Immunofluorescence pattern of DES and MYF5 in Gal-1-treated bfMSC cultures was similar to bfMSC exposed to 5-Aza (Fig. 5A and B). While DES immunofluorescence was widely distributed in the cytoplasm; MYF5 was compactly associated to the nucleus in some differentiated bfMSC. Immunofluorescence pattern of DES in SkGM-2 BulletKit-treated bfMSC cultures differed from other cultures and showed a punctuated pattern in distinctive cell aggregates (Fig. 5C). MYF5 was compactly associated to the nucleus in some differentiated bfMSC, as described for the other myogenic protocols.

\section{Discussion}

In the present study, bfMSC were isolated based on the capacity to adhere to plastic substrate under monolayer culture conditions from BM collected from abattoirderived bovine fetuses. Previously, we reported that the vast majority of the plastic-adherent population of 
(A)

1

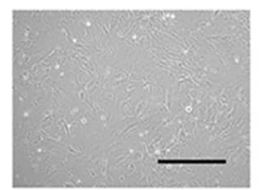

10

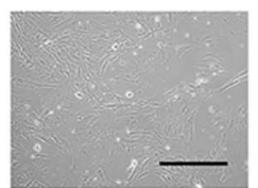

100

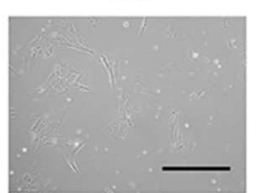

CON

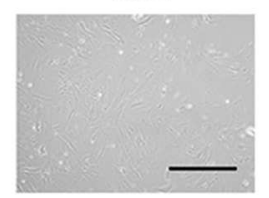

(B)

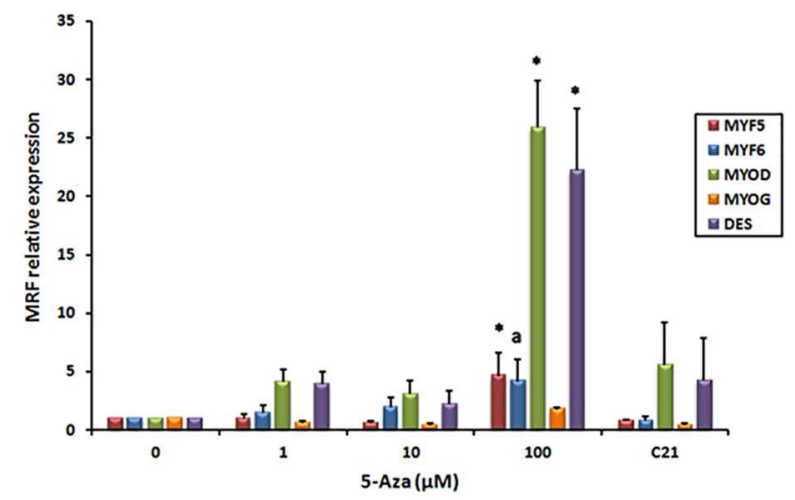

(C)

D0

D7

D14
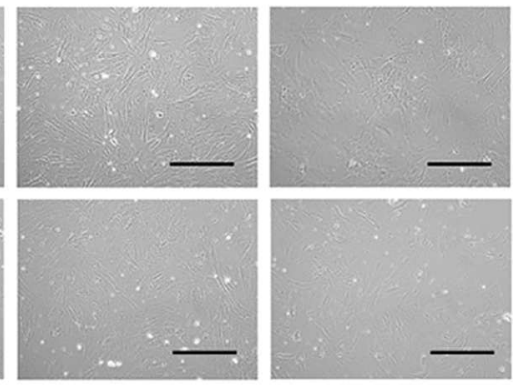

CON
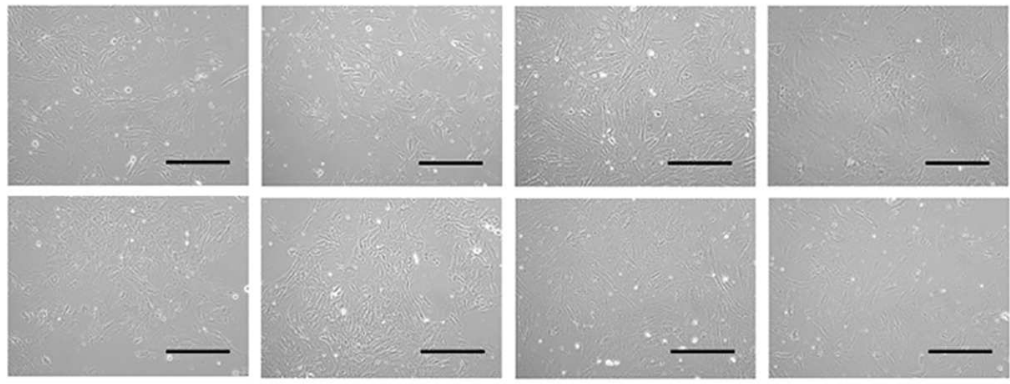

(D)

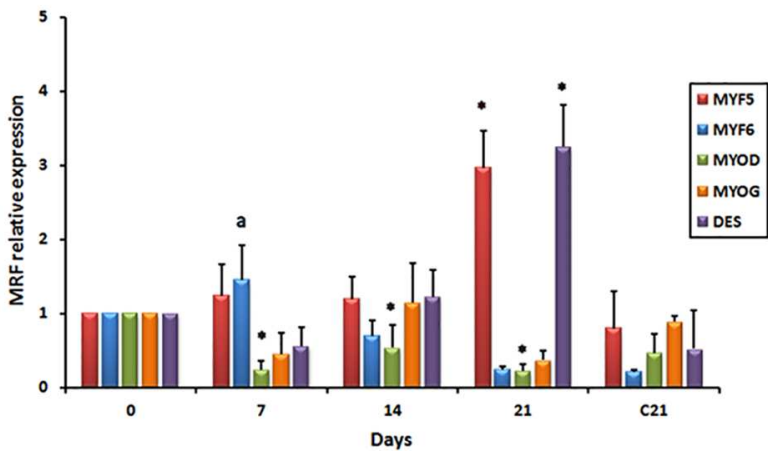

Figure 2. Myogenic in vitro differentiation of bfMSC under the effect of 5-Aza. (A) bfMSC exposed for 21 days to $100 \mu \mathrm{M}$ of 5 -Aza display higher mortality and lower plastic adherence compared to bfMSC cultured in presence of 1 and $10 \mu \mathrm{M}$ of 5-Aza. (B) MYF5, MYF6, MYOD, and DES mRNA levels were higher $(\mathrm{P}<0.05)$ in bfMSC cultured under $100 \mu \mathrm{M}$ of 5-Aza compared to other concentrations. (C) bfMSC cultured in presence of $10 \mu \mathrm{M}$ of 5-Aza displayed progressive cell morphology changes that included formation of cell projections and development of intricate cell interactions during the 21-day experiment. (D) While a decrease $(P<0.05)$ in levels of MYOD mRNA were detected (Days 7 to 21), an increment in levels of MYF6 (Day 7) and MYF5, and DES (Day 21) were observed in bfMSC exposed to $10 \mu \mathrm{M}$ of 5-Aza. $(*$ a) Indicate significant difference compared to Day 0 and Day 21 control (21C), respectively $(P<0.05)$. Scale bars: $500 \mu \mathrm{m}$. Abbreviations: DIF, Differentiation; CON, Control; C21, Negative Control at Day 21 of differentiation.

bfMSC expressed MSC specific markers CD29 and CD73 and lacked expression of hematopoietic surface markers CD34 and CD45 (18). These analyses and the multilineage potential indicate that plastic-adherent population of bfMSC fulfills the minimal criteria for definition of MSC. The simplicity of isolation and the potential to differentiate into several cell types lays the foundation for bfMSC, as an alternative source of MSC for research. In the present study, we first exposed bfMSC to a conventional method of cryopreservation 


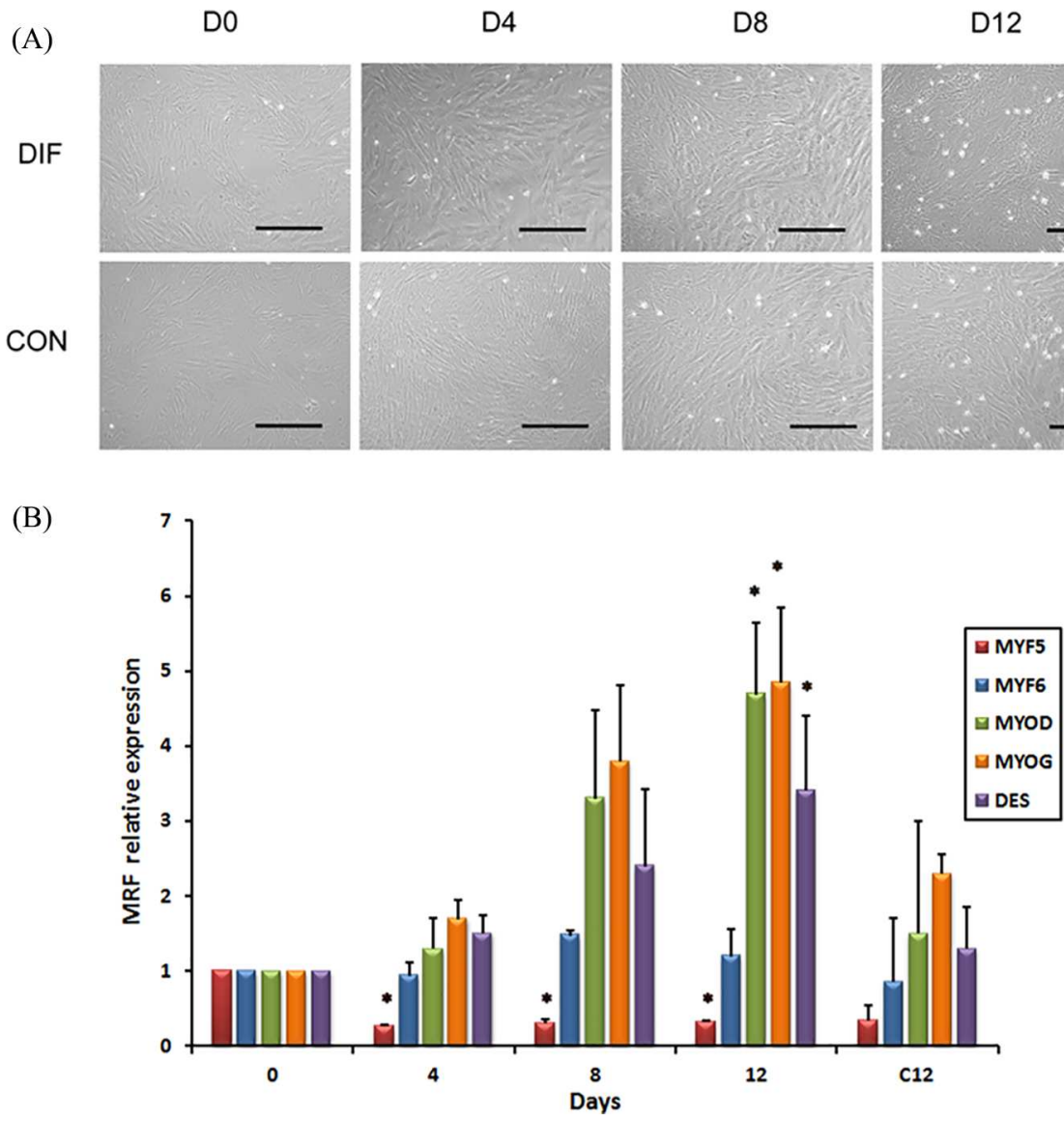

Figure 3. Myogenic in vitro differentiation of bfMSC under the effect of Gal-1. (A) GAL-1 induced organization of bfMSC into striated orientation pattern during a 12-day culture period. (B) A decreased ( $<0.05$ ) in MYF5 mRNA levels was detected (Days 7 to 21); whereas, an increase $(P<0.05)$ in MYOD, MYOG and DES was observed (Day 12) during bfMSC differentiation. $\left(^{*}\right)$ Indicate significant difference compared to Day 0 ( $P<0.05$ ). Scale bars: $500 \mu \mathrm{m}$. Abbreviations: DIF, Differentiation; CON, Control; C12, Negative Control at Day 12 of differentiation.

and determined a $>80 \%$ post-thaw viability. This value is similar to previously reported percentages in MSC from other species and tissue sources (21) and suggests the feasibility for cryopreservation and banking of bfMSC.

Considering that myogenic potential has been previously reported in MSC derived from human and murine sources $(22,23)$, these features make bfMSC potential candidates for the study of myogenesis and the defining mechanisms that regulate bovine muscle development. In the present study, we compared three protocols based on the use of 5-Aza, Gal-1, and SkGM-2 BulletKit for the in vitro myogenic differentiation of bfMSC.

Using dose-response experiments we found that cultures of bfMSC exposed to high concentrations of 5-Aza $(100 \mu \mathrm{M})$ increased MYF5, MYF6, MYOD, and DES mRNA levels but also augmented number of death cells. Furthermore, time-response experiments using a lower concentration of 5-Aza $(10 \mu \mathrm{M})$ indicated that bfMSC cultures increased MYF6, MYF5, and DES mRNA levels with no apparent effect on cells mortality. These results suggest that bfMSC subpopulations may respond differently depending on concentration of 5-Aza with either myogenic differentiation or cytotoxic effect. Despite the mechanistic effect has not been completely elucidated, treatment with 5-Aza has been successfully used for stimulation of myogenic differentiation in MSC derived from rat BM (6), human menstrual blood (24), and mouse dental pulp stem cells (7). However, other studies have reported low myogenic effect in human synovial membrane MSC (25) or massive cell death in hfMSC treated with 5-Aza (9). Thus, 5-Aza effect may also depend on the origin of MSC. We also found that demethylating effect of 5-Aza results in activation of a wide range of stage-specific MRF, including early (MYF5 and MYF6), intermediate (MYOD), and late $(D E S)$. Increase in $D E S$ expression is consistent with previous reports in rat MSC, where exposure to 5-Aza lead to its expression, via de stochastic hypomethylation of random DNA residues (6). Numerous transcriptional factors regulate gene expression by methylation of DNA 


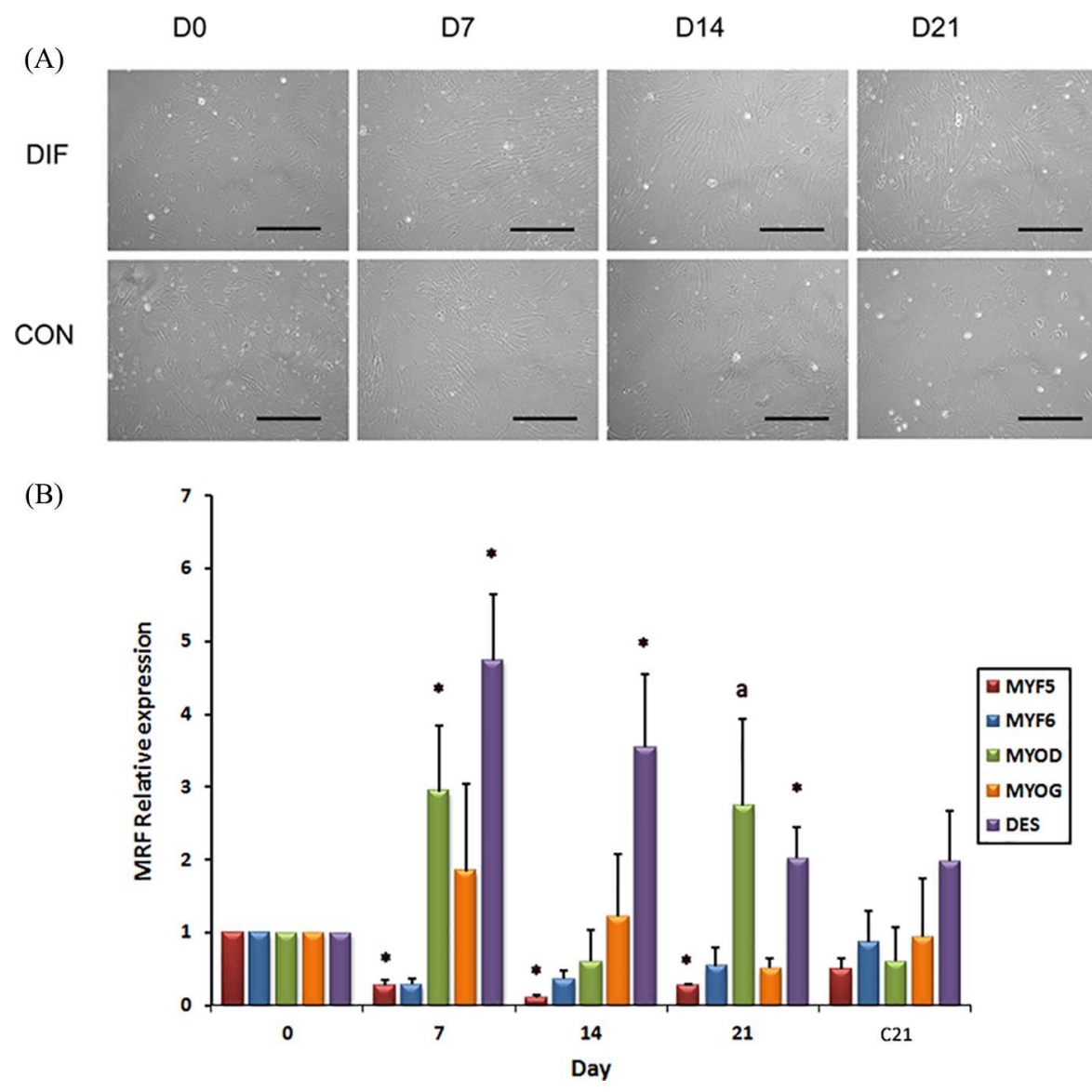

Figure 4. Myogenic in vitro differentiation of bfMSC under the effect of SkGM-2 BulletKit. (A) Subtle changes in cell morphology and distribution compared to bfMSC cultured in control media. (B) A decreased ( $P<0.05$ ) in MYF5 mRNA levels was detected (Days 7 to 21 ); whereas, an increase $(P<0.05)$ in MYOD (Days 7 and 21) and DES was observed (Days 7, 14 and 21) during bfMSC differentiation. (C) $\left({ }^{*}\right.$, a) Indicate significant difference compared to Day 0 and Day 21 control (C21), respectively $(P<0.05)$. Scale bars: $500 \mu m$. Abbreviations: DIF, Differentiation; CON, Control; C21, Negative Control at Day 21 of differentiation.

or methylation and/or acetylation of histones (26). We have previously reported that bfMSC increase DNA methyltransferases (DNMTs 1, 2, and 3), histone methylase (EZH2), and histone demethylase (KDM6B) during adipogenic differentiation (27). Moreover, bfMSC up-regulate KDM6B expression during osteogenic differentiation in association with the adoption of the osteogenic lineage (27). In myogenic studies, it has also been reported that muscle-specific transcriptional factors such as MYOD are inhibited by methylation of the transcriptional regulatory region (14) and, consequently, DNA demethylation by 5 -Aza treatment results in higher MYH expression and myotube formation in dental pulp stem cells (7). Thus, 5-Aza effect may result in demethylation of transcriptional factors involved in MSC myogenic differentiation; however, this effect is unspecific and may affect different regulatory regions of DNA depending on the level of methylation.

In our experiments, exposure of bfMSC to Gal-1 resulted in a high proportion $(>50 \%)$ of cells positive for DES after 12 days of culture. This proportion of cells may be comparable to previous reports, where $>60 \%$ of hfMSC exposed to Gal-1 expressed DES after the same culture period (8). We also detected that Gal-1-induced myogenic differentiation in bfMSC was accompanied by the sequential reduction in early MRF (MYF5) and increase in intermediate (MYOD) and late MRF (MYOG). Similarly, it was reported that hfMSC exposed to Gal-1 for 12 days, sequentially increase expression of MYF5, PAX7, MYOD and MYOG and differentiate in a stepwise manner into cells bearing a satellite cell immunophenotype $(\mathrm{PAX} 7+)$ and finally into multinucleated mature muscle fibers (8). Gal-1 is produced by myo blasts and plays a diverse variety of biological activities including cell adhesion, regulation of cell cycle and cell proliferation, immune functions and myogenesis (28). Despite role of Gal-1 in myogenesis is still under investigation, it has been proposed that Gal-1 block the interaction between laminin and its receptor in myoblasts $\alpha 7 \beta 1$ integrin, which results in inhibition of cell-extracellular matrix interaction and subsequent 


\section{(A) \\ Gal-1 \\ SkGM-2}

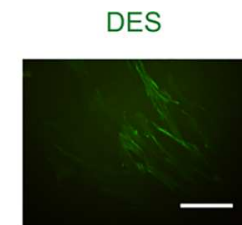

DES/DAPI
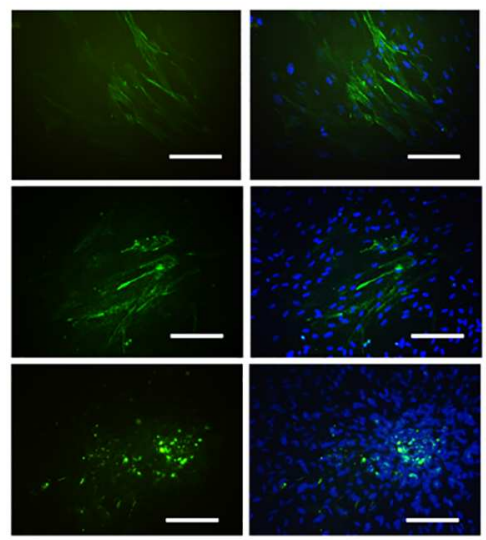

(B)
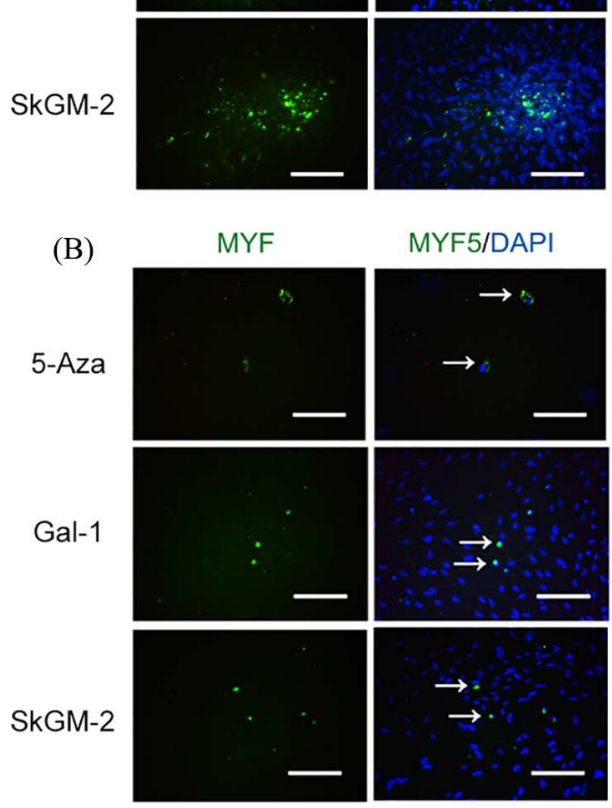

PC
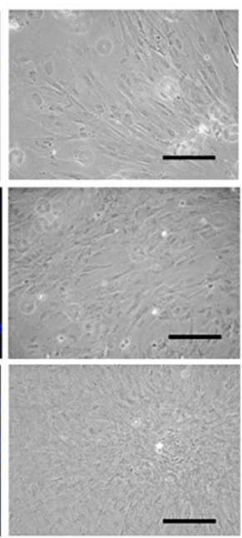

PC
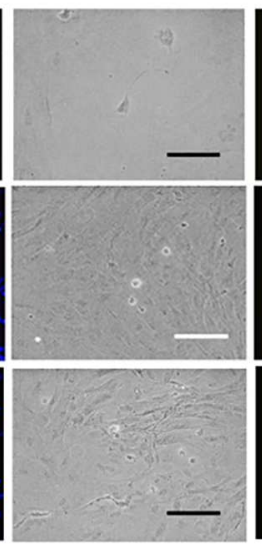

NC

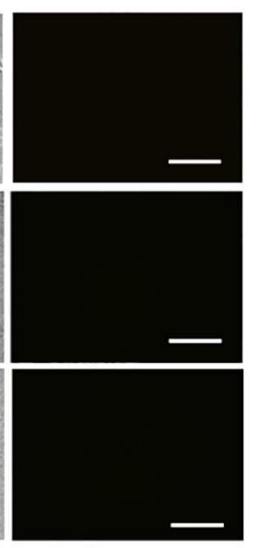

NC

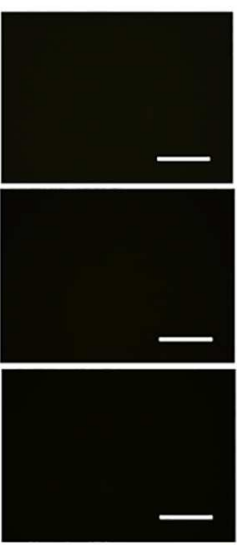

Figure 5. Immunodetection of DES and MYF5 in bfMSC after exposure to 5-Aza, Gal-1 or SkGM-2 BulletKit protocols of myogenic differentiation. While DES immunofluorescence was widely distributed in the cytoplasm (A); MYF5 was compactly associated to the nucleus in some differentiated bfMSC (B). DES and MYF5 (white arrows) were immunodetected in bfMSC exposed to $10 \mu \mathrm{M}$ of 5-Aza at Day 21 of culture, $100 \mathrm{nM}$ Gal-1 at Day 12 of culture or SkGM-2 Bullet Kit at Day 21 of culture. Abbreviations: PC, Phase Contrast; NC, Negative Control; DAPI, 4', 6-diamidino-2-phenylindole.

muscle cell differentiation (29). Gal-1 is highly expressed in adult BM-derived MSC and has been implicated as a soluble factor responsible for myogenic conversion of dermal fibroblasts $(30,31)$. Thus, the results of this study indicate that bfMSC exposed to Gal-1 undergone myogenic differentiation after a 12-day differentiation period and suggest that myogenic progression occurred from early (myoblast stage) to late differentiation (myocyte stage).

The commercial available SkGM-2 BulletKit was evaluated as myogenic inductor media in bfMSC during a 21-Day culture period. bfMSC exposed to SkGM-2 BulletKit reduced early MRF (MYF5) but increased intermediate (MYOD) and late MRF (DES) mRNA levels. We found one previous study that reported increased expression of MYH in human MSC after treatment with SkGM-2 BulletKit (9). Similar to our results, supplementation of dexamethasone has been associated to increased expression levels of MYOD and DES in human adipose and endometrial MSC
$(32,33)$. Glucocorticoids exert a wide range of effects in myoblast differentiation in vitro and dexamethasone has been associated to increased myotube fusion and terminal muscle differentiation in myocytes (34). Although we did not observe myotube formation, SkGM-2 BulletKit may induce myogenic effect by activating expression of muscle-specific genes, promoting myogenic progression from early (myoblast stage) to late differentiation (myocyte stage).

\section{Conclusion}

The protocols evaluated in bfMSC induced progress into myogenic differentiation until certain extent evidenced by changes in MRF gene expression. Biomarker expression profiles indicated that 5-Aza effect was not associated with sequential activation of MRF genes according to myogenic progression. In contrast, Gal-1 and SkGM-2 BulletKit induced sequential muscle-specific gene expression similar to myogenic progression from early 
(myoblast stage) to late differentiation (myocyte stage) during fetal bovine myogenesis. Moreover, the high percentage of post-thaw viability suggests the feasibility for cryopreservation and banking of bfMSC.

\section{Acknowledgments}

We thank Dr. Monica De Los Reyes from the Faculty of Veterinary Science at the University of Chile for the use of facilities at the Laboratory of Animal Reproduction.

\section{Funding}

This study was supported by grant 2014-69975 from the Office of Research and Development, University of Chile and grant Fondequip EQM120156 from the National Commission for Scientific and Technology Research (Conicyt) from the Ministry of Education, Government of Chile.

\section{Conflict of interest statement}

None of the authors has any financial or personal relationships that could inappropriately influence or bias the content of the paper.

\section{References}

1. Harichandan A, Bühring MJ. Prospective isolation of human MSC. Best Pract Res Clin Haematol 2011; 24:25-36.

2. Pittenger MF, Mackay AM, Beck SC, et al. Multilineage potential of adult human mesenchymal stem cells. Science 1999; 284:143-147.

3. Strioga M, Viswanathan S, Darinskas A, Slaby O, Michalek J. Same or not the same? Comparison of adipose tissue-derived versus bone marrow-derived mesenchymal stem and stromal cells Stem Cell Dev 2012; 21:2724-2752.

4. Gussoni E, Soneoka Y, Strickland CD, et al. Dystrophin expression in the $\mathrm{mdx}$ mouse restored by stem cell transplantation. Nature 1999; 401:390-394.

5. Muguruma Y, Reyes M, Nakamura Y, et al. In vivo and in vitro differentiation of myocytes from human bone marrow-derived multipotent progenitor cells. Exp Hematol 2003; 31:1323-1330.

6. Wakitani S, Saito T, Caplan AI. Myogenic cells derived from rat bone marrow mesenchymal stem cells exposed to 5-azacytidine. Muscle Nerve 1995; 18:1417-1426.

7. Nakatsuka R, Nozaki T, Uemura Y, et al. 5-Aza-2'deoxycytidine treatment induces skeletal myogenic differentiation of mouse dental pulp stem cells. Arch Oral Biol 2010; 55:350-357.

8. Chan J, O’Donoghue K, Gavina $M$, et al. Galectin-1 induces skeletal muscle differentiation in human fetal mesenchymal stem cells and increases muscle regeneration. Stem Cells 2006; 24:1879-1891.

9. Stern-Straeter J, Bonaterra GA, Juritz S, et al. Evaluation of the effects of different culture media on the myogenic differentiation potential of adipose tissue-or bone marrow-derived human mesenchymal stem cells. Int J Mol Med 2014; 33:160-170.
10. Megeney LA, Rudnicki MA. Determination versus differentiation and the MyoD family of transcription factors. Biochem Cell Biol 1995; 73:723-732.

11. Christ B, Brand-Saberi B. Limb muscle development. Int J Dev Biol 2002; 46:905-914.

12. Buonanno A, Apone L, Morasso MI, Beers R, Brenner HR, Eftimie R. The myoD family of myogenic factors is regulated by electrical activity: isolation and characterization of mouse MYF-5 cDNA. Nucleic Acids Res 1992; 20:539-544.

13. Halevy O, Novitch BG, Spicer DB, et al. Correlation of terminal cell cycle arrest of skeletal muscle with induction of p21 by MyoD. Science 1995; 267:1018-1021.

14. Mal AK. Histone methyltransferase Suv39h1 represses MyoD-stimulated myogenic differentiation. EMBOJ 2006; 25:3323-3334.

15. McDonald OG, Owens GK. Programming smooth muscle plasticity with chromatin dynamics. Circ Res 2007; 100:1428-1441.

16. Edmondson DG, Olson EN. A gene with homology to the myc similarity region of MyoD1 is expressed during myogenesis and is sufficient to activate the muscle differentiation program. Genes Dev 1990; 4:1450.

17. Shah SB, Davis J, Weisleder N, et al. Structural and functional roles of desmin in mouse skeletal muscle during passive deformation. Biophys J 2004; 86: 2993-3008.

18. Cortes Y, Ojeda M, Araya D, Dueñas F, Fernández MS, Peralta OA. Isolation and multilineage differentiation of bone marrow mesenchymal stem cells from abattoirderived bovine fetuses. BMC Vet Res 2013; 9:133.

19. Dueñas F, Becerra V, Cortes Y, et al. Hepatogenic and neurogenic differentiation of bone marrow mesenchymal stem cells from abattoir-derived bovine fetuses. BMC Vet Res 2014; 10:154.

20. Uezumi A, Ito T, Morikawa D, et al. Fibrosis and adipogenesis originate from a common mesenchymal progenitor in skeletal muscle. J Cell Sci 2011; 124:3654-3664.

21. Marquez-Curtis LA, Janowska-Wieczorek A, McGann LE, Elliot JAW. Mesenchymal stromal cells derived from various tissues: Biological, clinical and cryopreservation aspects. Cryobiology 2015; 71:181-197.

22. Zuk PA, Zhu M, Mizuno $\mathrm{H}$, et al. Multilineage cells from human adipose tissue: implications for cell-based therapies. Tissue Eng 2001; 7:211-228.

23. Dezawa $M$, Ishikawa $H$, Itokazu $Y$, et al. Bone marrow stromal cells generate muscle cells and repair muscle degeneration. Science 2005; 309:314-317.

24. Cui CH, Uyama T, Miyado K, Terai M, Kyo S, Kiyono T, Umezawa A. Menstrual blood-derived cells confer human dystrophin expression in the murine model of Duchenne muscular dystrophy via cell fusion and myogenic transdifferentiation. Mol Biol Cell 2007; 18:1586-1594.

25. De Bari C, Dell'Accio F, Tylzanowski P, Luyten FP. Multipotent mesenchymal stem cells from adult human synovial membrane. Arthritis Rheum 2001; 44: 1928-1942.

26. Ko M, Sohn DH, Chung H, Seong RH. Chromatin remodeling, development and disease. Mutat Res 2008; 647:59-67.

27. Díaz P, Cuevas F, Peralta OA. GFP labelling and epigenetic enzyme expression of bone marrow-derived 
mesenchymal stem cells from bovine foetuses. Res Vet Sci 2015; 99:120-128.

28. Watt DJ, Jones GE, Goldring $\mathrm{K}$. The involvement of galectin-1 in skeletal muscle determination, differentiation and regeneration. Glycoconj J 2004; 19:615-619.

29. Gu MJ, Wang WW, Song WK, Cooper DNW, Kaufman SJ. Selective modulation of the interaction of alpha 7 beta 1 integrin with fibronectin and laminin by L-14 lectin during skeletal muscle differentiation. J Cell Sci 1994; 107:175-181.

30. Goldring K, Jones GE, Sewry CA. The muscle-specific marker desmin is expressed in a proportion of human dermal fibroblasts after their exposure to galectin-1. Neuromuscul Disord 2002; 12:183-186.
31. Kadri T, Lataillade JJ, Doucet C. Proteomic study of Galectin-1 expression in human mesenchymal stem cells. Stem Cells Dev 2005; 14:204-212.

32. Rodriguez AM, Pisani D, Dechesne CA, et al. Transplantation of a multipotent cell population from human adipose tissue induces dystrophin expression in the immunocompetent mdx mouse. J Exp Med 2005; 201:1397-1405.

33. Tehrani HJ, Parivar K, Ai J, Kajbafzadeh A, Rahbarghazi R, Hashemi M, Sadeghizadeh M. Effect of dexamethasone, insulin and EGF on the myogenic potential on human endometrial stem cells. Iran J Pharm Res 2014; 13:659-664.

34. Belanto J, Diaz-Perez S, Magyar C, et al. Dexamethasone induces dysferlin in myoblasts and enhances their myogenic differentiation. Neuromuscul Disord 2010; 20:111-121. 